\title{
The Ring Imaging Cherenkov Detectors for LHCb
}

\author{
Antonis Papanestis* \\ CCLRC - RAL, Chilton, UK \\ on behalf of the LHCb RICH Collaboration \\ E-mail: a.papanestis@rl.ac.uk
}

The success of the LHCb experiment depends heavily on particle identification over the momentum range $2-100 \mathrm{GeV} / \mathrm{c}$. To meet this challenge, $\mathrm{LHCb}$ uses a Ring Imaging Cherenkov (RICH) system composed of two detectors with three radiators. $\mathrm{RICH} 1$ has both aerogel and gas $\left(\mathrm{C}_{4} \mathrm{~F}_{10}\right)$ radiators, while $\mathrm{RICH} 2$ has only a gas $\left(\mathrm{CF}_{4}\right)$ radiator. The design of $\mathrm{RICH} 1$ is almost complete, whereas RICH2 has been constructed and installed (Nov 2005).

Novel Hybrid Photon Detectors (HPDs) have been developed in collaboration with industry to detect the Cherenkov photons. A silicon pixel detector bump-bonded to a readout chip is encapsulated in a vacuum tube. A bi-alkali photocathode is deposited on the inside of the quartz entrance window to convert photons in the range $200-600 \mathrm{~nm}$. The pixel chip is manufactured in $0.25 \mu \mathrm{m}$ deep-submicron radiation-tolerant technology and consists of 1024 logical pixels, each pixel having an area of $0.5 \mathrm{~mm} \times 0.5 \mathrm{~mm}$. Photo-electrons are accelerated by a $20 \mathrm{kV}$ potential, resulting in a signal of typically 5000 electrons that is amplified and digitized at the LHC speed of $40 \mathrm{MHz}$. The HPDs are enclosed in iron shielding and Mumetal cylinders surround the individual detectors to protect them from magnetic fields within the external shielding of up to $50 \mathrm{mT}$. The mass production of a total of 484 HPDs required for the two RICH detectors has already commenced.

The design and current status of the LHCb RICH system will be reviewed. Results obtained using prototype HPDs to detect Cherenkov light in particle test beams using the full LHCb readout chain will be presented. Finally, the expected performance of the LHCb RICH system, obtained from realistic simulation, will be shown.

International Europhysics Conference on High Energy Physics

July 21st - 27th 2005

Lisboa, Portugal

${ }^{*}$ Speaker. 


\section{Introduction}

The LHCb experiment [1] is a single arm spectrometer with the purpose to study CP violation using B mesons produced at the LHC. The LHC will collide two beams of $7 \mathrm{TeV}$ protons. Under these conditions the production cross section of $b \bar{b}$ pairs is around $500 \mu \mathrm{b}$ and peaks at low polar angles with both quarks travelling in the same direction. As a result, the $\mathrm{LHCb}$ detector can acquire large numbers of B mesons with a vertical (non-bending) acceptance of $250 \mathrm{mrad}$ and horizontal (bending) acceptance of $300 \mathrm{mrad}$.

\section{The RICH system}

The requirements of particle identification in LHCb can be in seen in Figure 1, where the angle of the track is plotted against the momentum. The minimum momentum requirements (around $2 \mathrm{GeV}$ ) come from the momenta of kaons used for tagging the flavour of the $\mathrm{B}$ decays, while the high end of the spectrum $(100 \mathrm{GeV})$ is determined by two body decays of B mesons. It is clear from the graph that particle identification is not important for large polar angle tracks at high momentum. So a RICH system was designed with two RICH detectors and three radiators: silica aerogel, $\mathrm{C}_{4} \mathrm{~F}_{10}$ and $\mathrm{CF}_{4}$. The RICH1 detector provides particle identification for low momentum tracks for the full acceptance of the spectrometer, while RICH2 is used for high momentum tracks with a limited acceptance coverage (100 mrad vertical and $120 \mathrm{mrad}$ horizontal).

\subsection{RICH1}

The RICH1 detector (Figure 2) has two radiators, aerogel [2] and $\mathrm{C}_{4} \mathrm{~F}_{10}$. Cherenkov photons are focused by a set of spherical mirrors and reflected by a set of flat mirrors onto the photon detectors above and below the beam pipe. The photon detectors are placed inside two iron shielding boxes which serve two functions. Firstly the shielding directs the stray magnetic field into

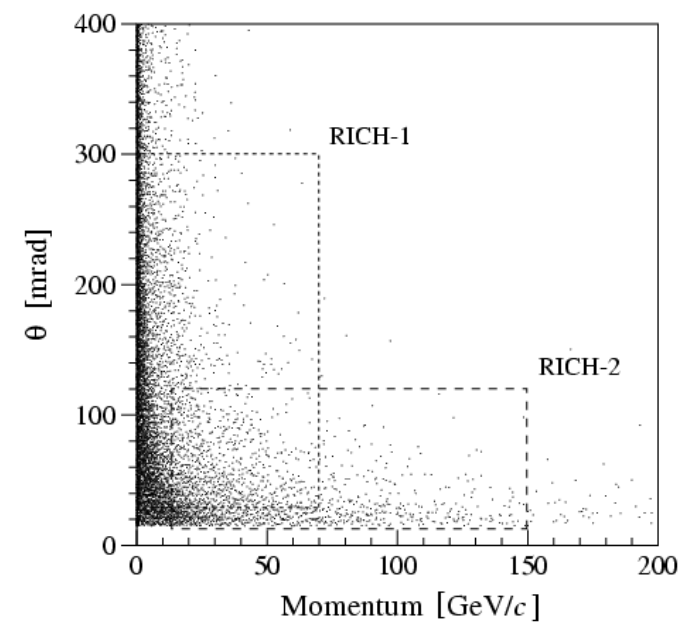

Figure 1: Polar angle versus momentum for all tracks in simulated $B_{d}^{0} \rightarrow \pi^{+} \pi^{-}$events. The regions of interest for RICH1 and RICH2 are indicated by the dashed lines.

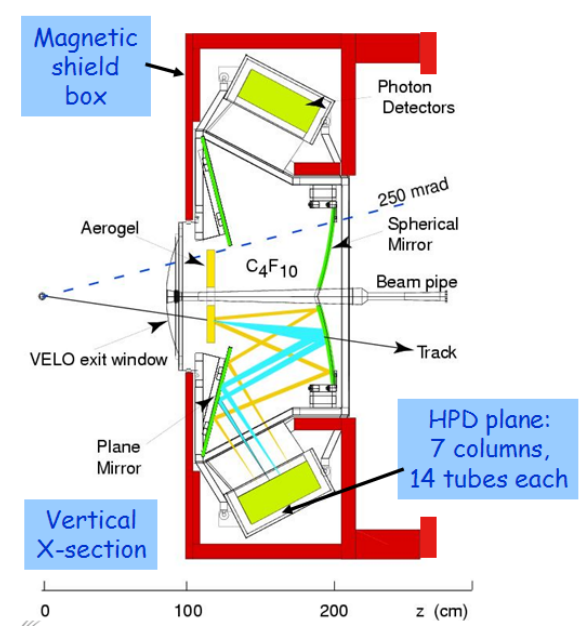

Figure 2: A schematic diagram of RICH1. The magnetic shielding ( 8 tons of Armco iron) has been carefully designed to guide the magnetic field away from the HPDs and towards the beam pipe. 
the RICH1 region for the purpose of the LHCb trigger [1]. Secondly the iron provides magnetic shielding for the RICH hybrid photon detectors (HPDs), which cannot operate in high magnetic fields.

The material of RICH1 inside the acceptance of the experiment has been kept to a minimum. The spherical mirrors are made of $4 \mathrm{~mm}$ of beryllium fused with a $0.5 \mathrm{~mm}$ layer of glass to provide the reflecting surface. There is no entry window as the detector is connected directly to the $\mathrm{LHCb}$ vertex detector (VELO), and the exit window is made of PMI foam sandwiched between $1 \mathrm{~mm}$ carbon fibre skins.

\subsection{RICH2}

RICH2 has a horizontal configuration rather than the vertical design as for RICH1 (Figure 3). The photon detectors are also enclosed in magnetic shielding and situated outside the acceptance. About 50\% more HPDs are required due to the bigger size of the detector. The entry window is constructed using the same design as the RICH1 exit window, with a $38 \mathrm{~mm}$ core due to the larger size. The exit window has the same core and $1 \mathrm{~mm}$ aluminium skins. The radiator is $\mathrm{CF}_{4}$ gas. The installation of mirrors in RICH2 is complete and the alignment and stability for the full set of mirrors in each half of the detector is $150 \mu \mathrm{rad}$.

\section{The Hybrid Photon Detector}

The HPDs [3], developed in collaboration with industry ${ }^{1}$, have been chosen for the $\mathrm{LHCb}$ RICH detectors. They are based on a silicon pixel detector bump-bonded to a binary $40 \mathrm{MHz}$ readout chip [4] and encapsulated in a vacuum tube with a quartz entrance window. A bi-alkali photocathode is deposited on the inside of the tube window, and the generated photo-electrons are focused on the pixel detector. The result is a photon detector with a pixel size of $2.5 \mathrm{~mm} \times 2.5 \mathrm{~mm}$ and excellent signal to noise ratio. The clear separation of the single photon signal peak to the noise peak allows the detector to operate in a binary mode with only $15 \%$ loss of efficiency. The total area covered by the HPDs is $2.6 \mathrm{~m}^{2}$, with an active coverage of $65 \%$. The photo-cathode is sensitive in wavelengths between 200 to $600 \mathrm{~nm}$ and the HPDs are radiation hard up to at least 30 kRad.

\section{RICH software and performance}

Detector simulation and performance evaluation has been an integral part of the RICH detector development. A complete simulation of the LHCb experiment, based on an object oriented framework [5] and GEANT4 [6], has been implemented, which allows the realistic evaluation of the RICH performance. Hits in the photon detectors are combined with tracks from charged particles to reconstruct the Cherenkov angle and a log likelihood method is used to calculate the most probable particle hypothesis.

The performance of the RICH system, using as criterion the identification of pions as light particles $(e, \mu, \pi)$ can be seen in Figure 4. The correct particle type is identified better than $80 \%$ over

\footnotetext{
${ }^{1}$ Delft Electronische Producten (DEP) - Photonis, The Netherlands, as the principal industrial partner.
} 


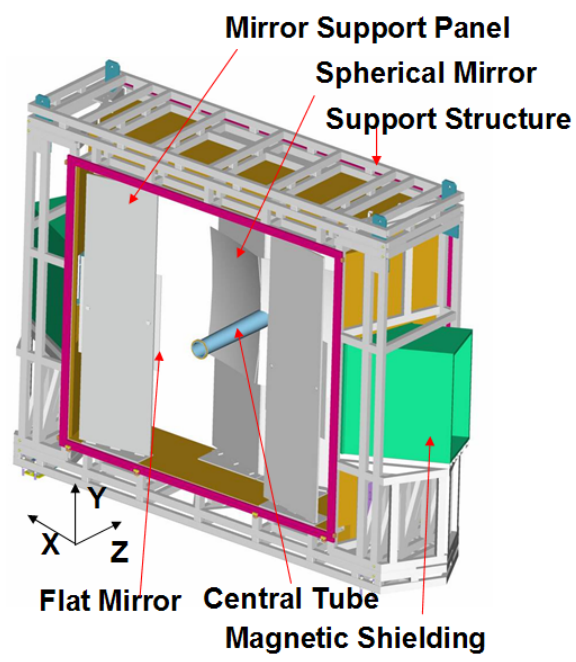

Figure 3: An engineering model of Rich2. The central tube connects the front and back windows (not visible) for extra stability.

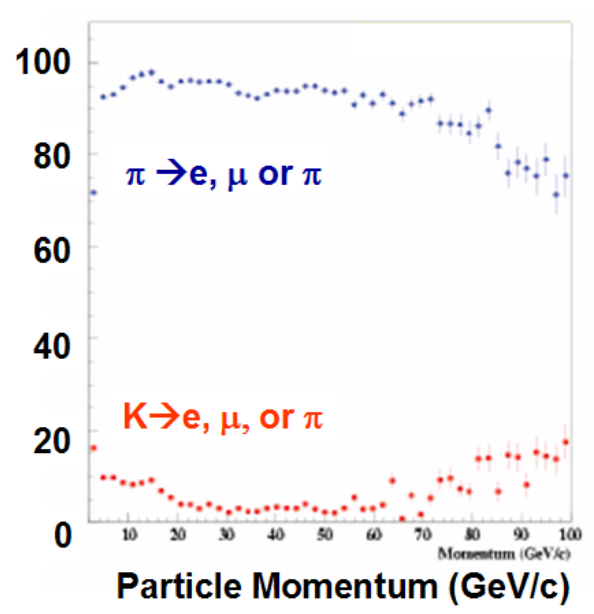

Figure 4: The performance of the RICH system when identifying pions as light particles versus momentum. The best performance is achieved between 20 and $60 \mathrm{GeV}$.

the momentum range $2-100 \mathrm{GeV}$, while the wrong hypothesis is assigned only at a few percent. The performance is very similar if the criterion is the identification of kaons as heavy $(K, p)$ particles. The reason for having the light and heavy description is that using information from other subdetectors of the experiment it is possible to make a better judgement and identify the exact particle type.

\section{Summary}

Particle identification is an important consideration in $\mathrm{LHCb}$, and the RICH system is a key component providing excellent pion and kaon identification. $\mathrm{RICH} 2$ has been constructed and is installed in the experimental hall. The engineering design for RICH1 has been completed and construction has started.

\section{References}

[1] The LHCb Collaboration, LHCb Re-Optimized Detector Design and Performance Technical Design Report, CERN/LHCC 2003-030, LHCb TDR9, September 2003.

[2] C. Matteuzzi, A RICH with aerogel for a hadron collider, Nucl. Instr. and Meth. A553 (356).

[3] M. Campbell et al., Performance of hybrid photon detector prototypes with encapsulated silicon pixel detector and readout for the RICH counters of LHCb, Nucl. Instr. and Meth. A504 (286).

[4] W. Snoeys, et al., Pixel readout electronics development for the ALICE pixel vertex and LHCb RICH detector Nucl. Instr. and Meth. A 465 (176).

[5] M. Cattaneo et al., Status of GAUDI event-processing framework, Proceedings of International Conference on Computing in High Energy Physics, Beijing, China, September 3-7, 2000.

[6] S. Agostinelli, et al., GEANT4 - a simulation toolkit, Nucl. Instr. and Meth. A $\mathbf{5 0 6}$ (250). 